\title{
Worth another look?
}

A paper that was published recently in Cancer Discovery indicates that patients with non-small-cell lung cancer (NSCLC) with tumours that have a specific subset of methylated genes might benefit from combined treatment with a low-dose DNA methyltransferase inhibitor and a histone deacetylase inhibitor (HDACI).

The use of DNA methyltransferase inhibitors in the clinic is not new: drugs such as azacitidine and decitabine are approved for the treatment of patients with myelodysplasia. Initially, these drugs were

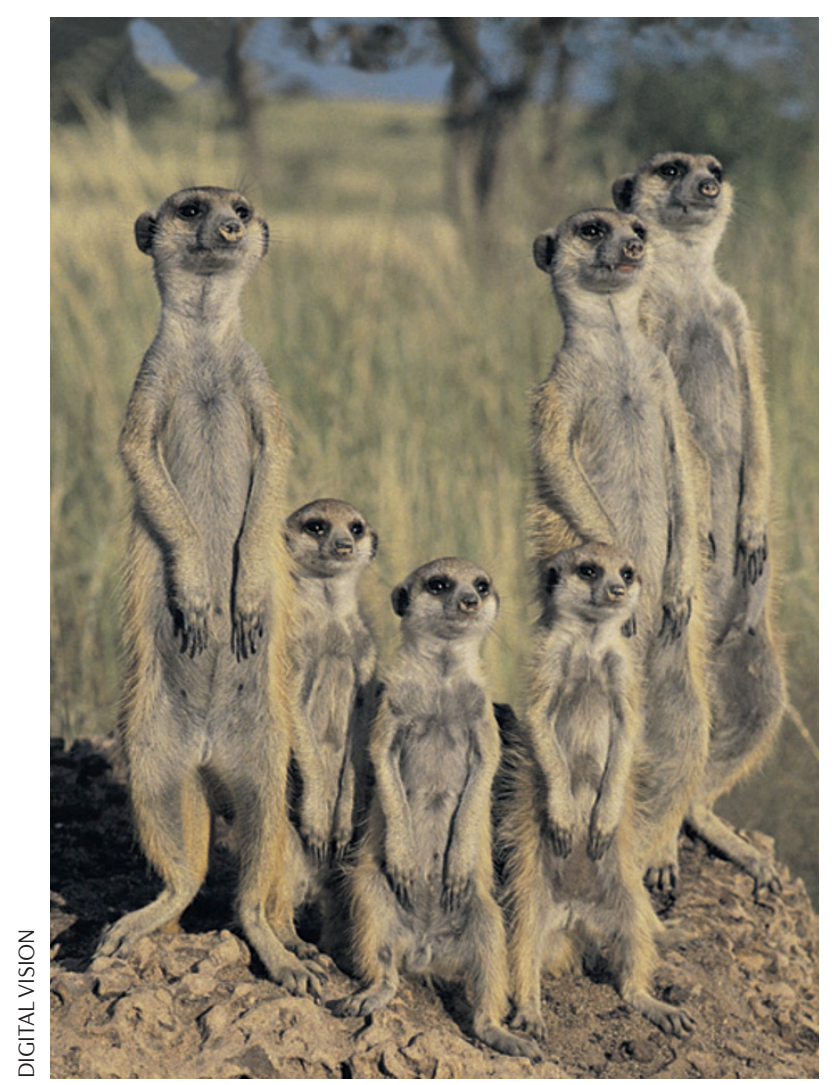

used at their maximum tolerated dose, at which they have a cytotoxic effect, but the side effects in patients with solid tumours were thought to be too severe for the minimal efficacy observed. However, the modulation of DNA methylation by these inhibitors is effective at lower doses that crucially retain efficacy in patients with myelodysplasia. HDACIs are also used in the clinic, and preclinical data indicate that a combination of a DNA methyltransferase inhibitor and an HDACI can lead to the re-expression of DNA-methylated genes.

Such findings have prompted Rosalyn Juergens and colleagues to look again at the use of DNA methyltransferase inhibitors in patients with solid tumours. Ten patients with heavily pretreated, refractory NSCLC were initially enrolled in the Phase I trial to determine the dose of azacitidine, and seven of these patients and an additional 35 similar patients enrolled into the Phase II trial received $40 \mathrm{mg}$ per $\mathrm{m}^{2}$ per day of azacitidine and $7 \mathrm{mg}$ of entinostat (an HDACI) on days 3 and 10 of a 28 -day cycle. One patient had a complete response of 14 months duration and another patient had a partial response that lasted 8 months. Ten patients had stable disease and 22 had progressive disease after two cycles of treatment. The 11 remaining patients could not be evaluated as they did not complete one cycle of therapy. In an intent to treat analysis of all the patients enrolled, median progression-free survival was 7.4 weeks (95\% confidence interval (CI) of 7-8 weeks) and median overall survival was 6.4 months (95\% CI of
3.8-9.2 months) - results which compare favourably with existing therapeutic options.

Interestingly, the authors also looked at the responders and nonresponders in terms of gene methylation status. Methylation of any two of four genes - APC, RASSF1A, $C D K N 2 A$ and $C D H 13$ - in the tumour and draining lymph nodes is predictive of a poor prognosis in patients with stage I NSCLC. Promoter methylation status of these four genes before and after one cycle of treatment was examined in the 26 patients who had sufficient levels of circulating free DNA. Ten patients had two genes that were methylated before treatment and showed a decrease in the methylation of these after the first cycle. Importantly, eight of these ten patients had either stable disease or objective responses. Of the remaining 16 patients who did not show changes in DNA methylation of these genes, only four had stable disease and there were no objective responses.

Larger trials will be needed to establish whether such epigenetic therapies are only efficacious in patients with a DNA methylation signature. However, given that four of 19 patients had objective responses to subsequent cancer therapies, there is some initial evidence that this therapy could sensitize tumours to subsequent cancer treatments. Nicola McCarthy

ORIGINAL RESEARCH PAPER Juergens, R. A. et al. Combination epigenetic therapy has efficacy in patients with refractory advanced non small cell lung cancer. Cancer Discovery 9 Nov 2011 (doi:10.1158/2159-8290.CD-11-0214) 